\title{
Carta del Presidente del CACI
}

\author{
Letter from the President of $\mathrm{CACl}$
}

Revista Argentina de Cardioangiología Intervencionista 2018;9(1):63-64. Doi: 10.30567/RACI/201801/0063-0064

Estimados colegas y amigos, es para mí una gran satisfacción ponerme en contacto con ustedes a través de este nuevo número de nuestra revista.

Quisiera aprovechar la oportunidad para compartir algunos de los objetivos que esta Comisión Directiva 2018-2019, que tengo el honor de presidir, ha planteado para esta gestión:

- Fortalecer los lazos con el Ministerio de Salud de la Nación, con ANMAT, otras autoridades gubernamentales y financiadores.

- Reforzar el aspecto gremial del CACI.

- Mantener en vigencia el subsidio por enfermedad o accidente creado por resolución de Comisión Directiva 2016-2017.

- Mantener fluidos canales de comunicación con los socios.

- Afianzar las relaciones con las sociedades científicas nacionales e internacionales, especialmente fortalecer aún más la relación con SOLACI.

- Fortalecer aún más el crecimiento de nuestra revista, invitando a todos ustedes a enviar trabajos científicos originales y casos clínicos, que por su originalidad y complejidad sean de interés para la comunidad cardiológica.

- Ratificar el compromiso de participación en el programa Stent Save a Life.

- Promover y poner a disposición de los socios modernos instrumentos de educación médica continua.

Quisiera detenerme en este último punto y expresarles que, con gran satisfacción y entusiasmo, celebro el lanzamiento del tomo I del E-Book de Hemodinamia y Cardioangiología Intervencionista.

Este instrumento de educación médica continua es el resultado de 28 años de experiencia de los integrantes del Área de Docencia del CACI, quienes han formado a través de la Carrera UBA-CACI de Hemodinamia, Angiografía general y Cardiangiología Intervencionista y el Programa de actualización en Hemodinamia y Cardioangiología Intervencionista al 90\% de los miembros activos de nuestro colegio.

El e-book, entre otras particularidades, nos proporciona acceso a una actualización continua en nuestra propia lengua.

En los últimos años, hemos sido testigos de la incesante incorporación de recursos de extrema utilidad para la enseñanza de la medicina, y en nuestro caso, de la Cardioangiología Intervencionista.

A través de los cinco tomos del E-Book de Hemodinamia y Cardioangiología Intervencionista se desarrollan todos los temas actuales de nuestra especialidad.

Pueden estar seguros, los autores y miembros del Área de Docencia del CACI, que a través de esta nueva herramienta educativa están contribuyendo en gran medida con las necesidades de actualización de sus colegas.

No puedo dejar de mencionar que el lanzamiento del primer tomo de esta innovadora herramienta de actualización se produce simultáneamente con otro paso fundamental de nuestro Colegio en dirección a brindar modernos instrumentos de educación y entrenamiento a nuestros socios, con la ampliación de nuestra sede, al concretarse recientemente, en diciembre de 2017, la incorporación de un nuevo piso, que se destinará fundamentalmente a la instalación de un Centro de Simulación, donde contaremos con simuladores de última generación.

En este Centro de Simulación del CACI, no solo realizarán sus prácticas y entrenamientos los alumnos de la Carrera UBA-CACI y los del Curso de Actualización CACI, sino que se desarrollarán cursos para médicos de nuestro país como del resto de Latinoamérica.

El uso de la simulación en Cardioangiología Intervencionista no solo es esencial para la formación de los 
nuevos especialistas, sino que probablemente asumirá un papel cada vez mayor en el mantenimiento de la certificación para especialistas con experiencia.

De esta forma, uno de los principales objetivos de nuestro Colegio, en su compromiso de ofrecer nuevos proyectos y posibilidades educativas a sus socios, se ve materializado a través de dos propuestas innovadoras:

- el E-Book de Hemodinamia y Cardioangiología Intervencionista,

- el Centro de Simulación CACI.

Dr. Aníbal Damonte

Presidente del CACI 